\title{
OPINIONES DE LOS COSTARRICENSES ACERCA DEL ESPAÑOL GENERAL, LA CORRECCIÓN LINGÜÍSTICA, EL ESPAÑOL EN LOS MEDIOS DE COMUNICACIÓN Y EN LA EDUCACIÓN
}

\author{
Annette Calvo Shadid \\ Jacqueline Castillo Rivas
}

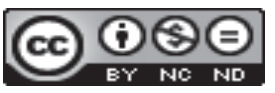

Esta obra está bajo una licencia Creative Commons

Reconocimiento-No Comercial-Sin Obra Derivada 



\title{
OPINIONES DE LOS COSTARRICENSES ACERCA DEL ESPAÑOL GENERAL, LA CORRECCIÓN LINGÜÍSTICA, EL ESPAÑOL EN LOS MEDIOS DE COMUNICACIÓN Y EN LA EDUCACIÓN
}

\author{
OPINIONS BY COSTA RICANS ABOUT GENERAL SPANISH, \\ LINGUISTIC CORRECTION, AND SPANISH IN THE MEDIA AND \\ EDUCATION
}

\author{
Annette Calvo Shadid \\ Jacqueline Castillo Rivas
}

\begin{abstract}
RESUMEN
En este estudio se recogen los datos de 404 entrevistas acerca de opiniones de los habitantes de la ciudad de San José sobre la corrección lingüística, la unidad lingüística, el español en los otros países de habla hispana, actitudes afectivas por las distintas variedades dialectales, sobre el español en los medios de difusión como el cine, la televisión, la radio, y el de la información telefónica. Se reflexiona, además, sobre el futuro de la lengua española y sobre la unidad y la heterogeneidad del español.
\end{abstract}

Palabras clave: actitudes lingüísticas, sociolingüística, español de Costa Rica, unidad y heterogeneidad de la lengua española.

\begin{abstract}
In this study, data from 404 interviews about opinions of the inhabitants of San Jose City on linguistic correctness, linguistic unity, Spanish in other Spanish-speaking countries, affective attitudes by different dialects are collected on Spanish in the media such as movies, television, radio, and telephone information. It also thinks about the future of the Spanish language and the unity and diversity of Spanish.

Key words: linguistic attitudes, sociolinguistics, Costa Rican Spanish, unity and diversity of Spanish language.
\end{abstract}

Dra. Annette Calvo Shadid. Universidad de Costa Rica. Profesora catedrática. Escuela de Filología, Lingüística y Literatura. Decana de la Facultad de Letras. Costa Rica.

M.Sc. Jacqueline Castillo Rivas. Universidad de Costa Rica. Profesora Catedrática. Facultad de Odontología. Costa Rica.

Correo electrónico: acalsha@yahoo.com.mx

jac9701@yahoo.com

Recepción: 21- 02- 2014

Aceptación: 18- 03- 2014 


\section{Introducción}

\subsection{El español del futuro o el futuro del español}

En un artículo de López-Morales (2005) titulado "El futuro del español", el autor habla de la preocupación de muchos sobre el español llamado "neutro" que se ha posicionado en los medios de comunicación, un español que "homogeniza" al máximo las variedades dialectales, en el que desaparecen los rasgos definitorios de las características culturales que esas variedades conllevan. Al respecto, el autor señala: "Se piensa que la buscada neutralidad se consigue simplificando la lengua y reduciendo el vocabulario a mínimos insospechados, es decir, a costa de trabajar con una modalidad desleída, raquítica y despersonalizada, y se culpa de este hecho a los resultados de la globalización lingüística que estamos sufriendo" (LópezMorales, 2005, p. 479).

A propósito de este y otros temas, ¿qué opinan los costarricenses respecto de la "corrección lingüística", de la llamada "unidad lingüística", del español de las diversas naciones, y cuáles son sus preferencias dialectales en el español de los medios de difusión y en la educación? ¿Será expansiva la onda del "español neutro"? Estas preguntas son respondidas a continuación por los hablantes del cantón de San José, en un estudio de 404 muestras de opinión recogidas por Calvo Shadid y Castillo-Rivas (2013). ${ }^{1}$

\subsection{Sobre el español correcto, la unidad del español y el español en los medios de comunicación}

Sobre el español correcto se han escrito numerosas obras; por mencionar solo algunas de ellas se encuentran el Diccionario Panhispánico de Dudas (2005), la Guía práctica del español correcto (2009), El libro del español correcto (2012), El buen uso del español (2013) y Las 500 dudas más frecuentes del español (2013) las cuales persiguen que los hablantes se expresen "bien" de forma oral y escrita; son libros para el gran público y, unos más, otros menos, son de carácter prescriptivo o normativo; no son exhaustivos porque, como opina acertadamente Martínez de Sousa (2005, p. 1), si se pretende "responder a todas las dudas existentes y prever las que están por venir, el fracaso en el empeño será absoluto porque tal obra no es posible". El autor habla específicamente del DPD, pero lo mismo se podría decir del resto de las obras sobre el español correcto.

Acerca de la unidad del idioma, este es un tema tratado abundantemente en la lingüística hispánica. ${ }^{2}$ Desde Andrés Bello, ${ }^{3}$ uno de los motivos que impulsó su gramática fue el interés del autor de preservar la unidad del español. Dicha unidad se dirige de nuevo al tema de la corrección. Wagner (2006) ${ }^{4}$ señala: "En las Nociones preliminares de su Gramática define ésta como el "arte de hablar correctamente, esto es, conforme al buen uso, que es el de la gente educada [...], porque es el más uniforme, en las varias provincias y pueblos que hablan una misma lengua, y por lo tanto el que hace que más fácil y generalmente se entienda lo que se dice [...]" (1954, p. 25)". En esta cita se observa cómo el mismo Bello se aparta de "lo que él llama 'supersticioso casticismo' de las gramáticas españolas, que rechazaban como 'viciosa' cualquier expresión americana que no se practicara en la península” (Wagner, 2006).

Por su parte, Coseriu (1990) señala que toda actividad en pro de la unidad de la lengua es indudablemente una actividad de tipo político, pero la naturaleza de la actividad es tarea de la teoría lingüística. El autor habla de enfoques erróneos, como aquellos conservadores 
y puristas que pretenden eliminar los usos locales y que tienden a reducir lo correcto a lo "ejemplar" (el español de España, por lo general). Por el contrario, los liberales y tolerantes reducen todo a señalar que todo uso es bueno y aceptable "con tal que se entienda".

El autor señala que "hablar español" no se refiere a un solo modo de hablar, sino a muchos, que corresponden a distintos discursos. La unidad se trata del uso de una lengua común, que coincide en su extensión con el ámbito de la lengua histórica; es estándar o pauta de referencia para las variedades regionales y es la que representa a la lengua histórica en el plano interidiomático e internacional. Además, la unidad trata más sobre ejemplaridad que sobre corrección. Es el modelo, el ideal de lengua común, la cual tiene un prestigio, un valor sociocultural. En este sentido, tanto el español de América como el de España son español, el de América tan "legítimo" y "auténtico" como el otro, señala el autor (Coseriu, 1990, p. 62). Ambas variedades deben confrontarse como tradiciones equivalentes y de igual jerarquía; es decir, con tradiciones diferentes pero igualmente genuinas. Agrega que no es razonable aspirar a una homogeneidad y fijeza total en el plano de las normas y aboga por una unidad del idioma como ejemplaridad panhispánica que sea dinámica, flexible y política y culturalmente "oportuna".

López-Morales (2005) recalca que la unidad del español no está amenazada: "En realidad, no hay que albergar tales temores, puesto que estamos ante una lengua «relativamente » homogénea, circunstancia esta que no ha frenado su expansión más allá de sus fronteras naturales" (López-Morales, 2005, p. 477), e incluye una nota sociolingüística respecto de esta homogeneidad al recalcar:

\footnotetext{
Es verdad que entre hablantes de todo el mundo hispánico y, en general, entre los que manejan un español aprendido, la comunicación suele ser muy transparente, sobre todo si pertenecen a niveles altos y medio-altos del espectro sociocultural. No ocurre exactamente lo mismo, sin embargo, cuando es otro el estatus social de los Es verdad que entre hablantes de todo el mundo hispánico y, en general, entre los que manejan un español aprendido, la comunicación suele ser muy transparente, sobre todo si pertenecen a niveles altos y medio-altos del espectro sociocultural. No ocurre exactamente lo mismo, sin embargo, cuando es otro el estatus social de los hablantes. Hoy, las investigaciones sociolingüísticas dejan muy claro la constante relación asociativa existente entre niveles medios y bajos y una creciente presencia de peculiaridades lingüísticas, y no solo léxicas. (López-Morales, 2005, pp. 477-478)
}

Sobre el español en los medios de comunicación, López-Morales (2005) se refiere también al extraordinario desarrollo del idioma en ellos y cómo eso, precisamente, es lo que podría crear una tendencia a homogeneizar demasiado el idioma, a fuerza de intentar un artificial español "neutro": "Lo que preocupa a muchos [...] es que la necesidad de homogeneizar al máximo nuestras variedades dialectales fuerce a crear un «español neutro» (etiqueta empleada con fuertes matices peyorativos), en el que desaparezcan los rasgos definitorios de la personalidad cultural que esas variedades conllevan" (López-Morales, 2005, p. 479).

\section{Análisis de los datos}

\subsection{El español general: Opiniones sobre la corrección lingüística}

\section{¿Qué entiende usted por "hablar correctamente”?}

Ante esta pregunta, el 39\% de los entrevistados considera que hablar correctamente se relaciona con la pronunciación y con el acento, seguidos del uso adecuado de las palabras. Se encontró diferencia estadísticamente significativa por nivel educativo $(\mathrm{p}=0,007)$ y por nivel 
socioeconómico $(\mathrm{p}=0,003)$. Esto se debe a que las personas de nivel socioeconómico alto y las que tienen educación universitaria, indican que hablar correctamente es el uso adecuado de las palabras, seguido de la demostración de educación y cultura y, finalmente, el acento. Las personas con niveles socioeconómicos bajo y medio, así como aquellas que tienen nivel educativo de primaria o secundaria, indican que la mayor importancia la tiene el acento.

\section{Gráfico 1}

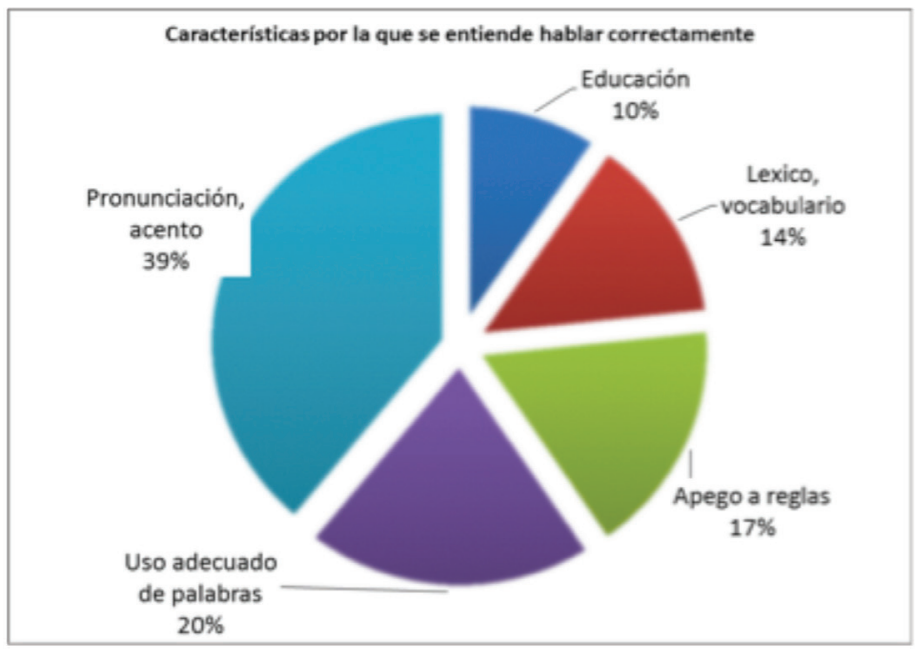

¿Cuán importante considera usted hablar correctamente?

El 99\% de los entrevistados considera importante hablar correctamente, sin que se encontrara diferencia estadísticamente significativa por edad $(\mathrm{p}=0,727)$, nivel socioeconómico $(p=0,323)$ y nivel educativo $(p=0,656)$. Por sexo se encontró diferencia estadísticamente significativa $(\mathrm{p}=0,018)$ debido a que un mayor porcentaje de mujeres considera muy importante hablar correctamente con respecto al observado en los hombres.

\section{Gráfico 2}

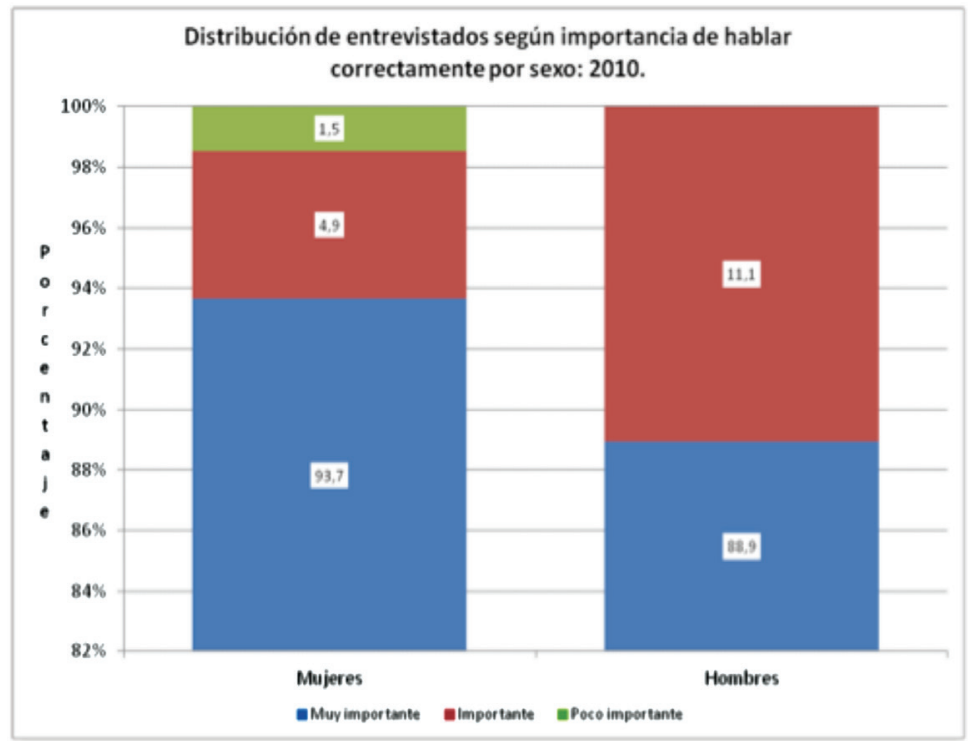


En cuanto a los porcentajes, es interesante señalar que los rubros "muy importante" e "importante" en los hombres comprende el 100\%, mientras que en las mujeres hay un pequeño porcentaje que manifiesta que es "poco importante" el cual, sin embargo, es el que explica la diferencia estadísticamente significativa. Es decir, hay mujeres (a diferencia de los hombres, para quienes en su totalidad, hablar correctamente es importante) que actualmente consideran que hablar correctamente no es tan necesario. Por tanto, hay que empezar a colocar "con alfileres" los resultados que menciona López-Morales (2004) con referencia a la variable sexo/ género: "los datos acumulados hasta la fecha demuestran que las mujeres son más conscientes de la valoración que su comunidad hace de los fenómenos del lenguaje, y apoyan aquellos que obtienen más alto estatus en la evaluación social.

Por otro lado, entre las razones que establecen los entrevistados, indican que hablar correctamente es (muy) importante para comunicarse (36\%), entender lo que se dice (26\%), relacionarse (21\%) y demostrar la cultura y educación (15\%). El 100\% de los entrevistados considera muy importante o importante que se les entienda aunque "hable con errores".

Mencione un país de habla hispana en el cual, desde su punto de vista, se hable más "correctamente" el español.

El 53\% de los entrevistados opina que el país donde se habla más correctamente es España, seguido de Costa Rica (16\%), Colombia (9\%) y Chile (5\%); el 12\% restante indica principalmente países de Suramérica y un $5 \%$ indica que ningún país.

\section{Gráfico 3}

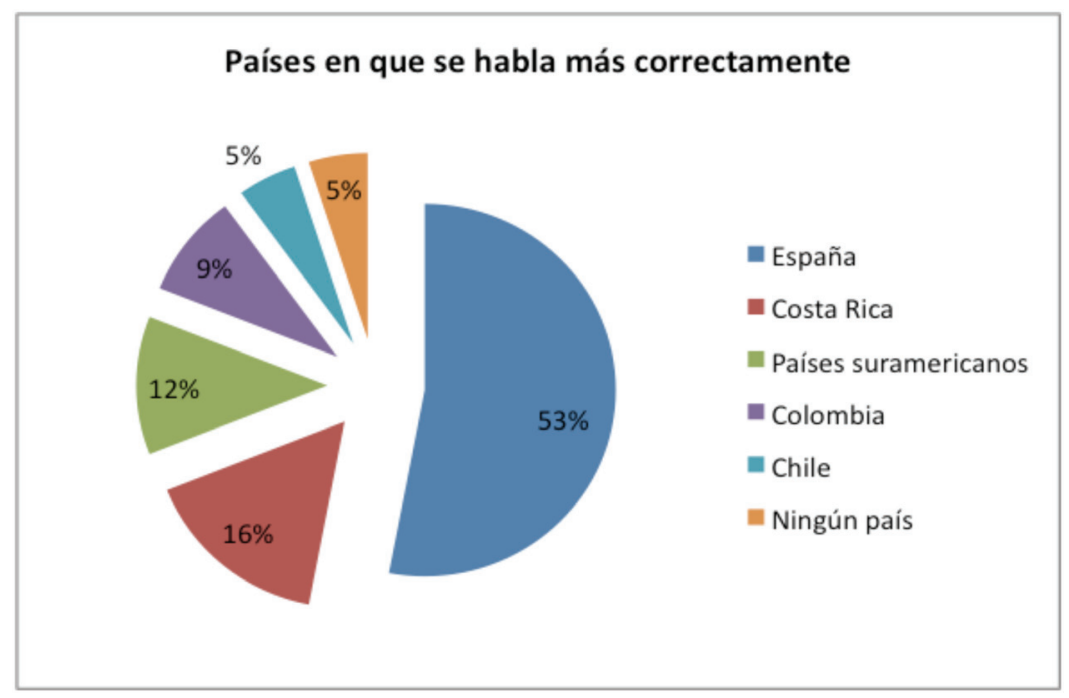

Se encontró diferencia estadísticamente significativa por nivel socioeconómico $(\mathrm{p}=0,0014)$ y por nivel educativo $(\mathrm{p}=0,002)$. Las personas con nivel socioeconómico más alto y las de nivel educativo universitario muestran una diferencia, pues la proporción de personas de este grupo que opina que España es el país donde se habla más correctamente el español disminuye a un $43 \%$, mientras que países como Colombia y Chile, y países del Cono Sur, aumentan a porcentajes mayores al $15 \%$.

En cuanto a las razones por las cuales se considera que en esos países se habla más correctamente el español se encuentran "porque España es la cuna del idioma”, seguido por la 
categoría de "porque hablan bien" y por el "acento". Se encontró diferencia estadísticamente significativa por sexo $(\mathrm{p}=0,030)$, ya que las mujeres se inclinan a señalar que en un país se habla correctamente porque "se entiende", mientras que los hombres le dieron más importancia al "acento".

Mencione un país de habla hispana en el cual, desde su punto de vista, se hable "incorrectamente" el español.

Nicaragua es el país en donde se considera que hablan más incorrectamente (42\%), seguido de México (8\%), Puerto Rico (7\%) y se tiene un $43 \%$ de los demás países. Se encontró diferencia estadísticamente significativa según nivel educativo $(\mathrm{p}=0,000)$ y nivel socioeconómico, cuya diferencia radica en que entre las personas de educación universitaria y las que tienen nivel socioeconómico alto, disminuye la proporción de personas que opina que es Nicaragua donde se habla más incorrectamente; se distribuye más homogéneamente entre todos los países y se incrementa la proporción de personas que indica "en ningún país".

Gráfico 4

\section{Países en los que se habla más incorrectamente}

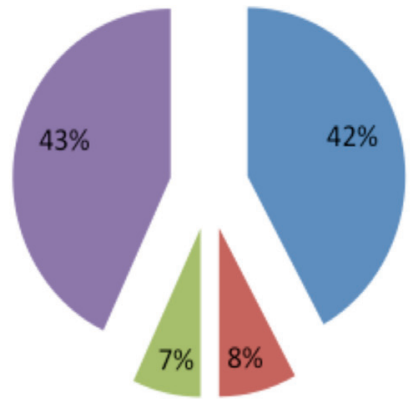

Nicaragua

- México

Puerto Rico

Otros países

Respecto de la opinión de por qué consideran que en ese país se habla más incorrectamente, la primera opinión fue debido al "acento" (28\%); hablan mal en cuanto al uso de palabras inapropiadas o al uso de dichos (15\%); el cambio de significado de las palabras (15\%) y la cultura y educación (14\%).

\subsection{Opiniones acerca de la unidad lingüística}

¿Sería bueno que todos habláramos el mismo español (en los países donde se habla)?

En cuanto a unidad lingüística, el $70 \%$ de los entrevistados indica que sería bueno que todos hablaran la misma variedad. La proporción de personas mayores de 55 años considera con mayor frecuencia que es mejor la unidad lingüística (80\%).

Se encontró diferencia estadísticamente significativa en la proporción de personas que indican que debe ser el mismo castellano. El grupo de personas con primaria y secundaria (70\%) es mayor con respecto al observado en las personas con educación universitaria (30\%). 


\section{Gráfico 5}

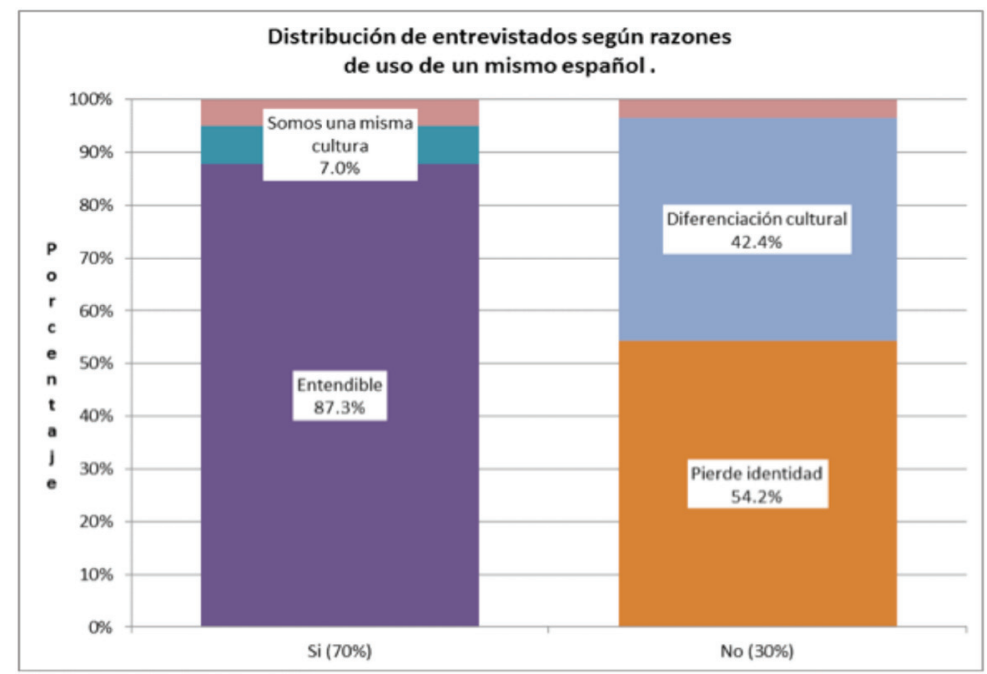

Entre las razones por las que se considera que debería haber una sola variedad, el $88 \%$ indica que es para poderse entender mejor, mientras que de las personas que están en contra, el $55 \%$ indica que se perderían la cultura y la idiosincrasia de cada país y el $45 \%$ indica que esto permite la diferenciación cultural.

Si todos tuviéramos que hablar el mismo español, ¿el de qué país le gustaría que fuera?

Si se tuviera que hablar el mismo español, el $55 \%$ de los entrevistados indica que escogería el de Costa Rica, el 26\% el de España, el 7\% el de Colombia y un 4\% el de Argentina; se encontró diferencia estadísticamente significativa por nivel educativo $(\mathrm{p}=0,003)$ debido a que la proporción de personas que indica que escogería el español de Costa Rica se incrementa al $67 \%$ en los niveles de primaria y secundaria, y disminuye a un $11 \%$ el español que se habla en España. Las personas con educación universitaria tienden a escoger más todos los países.

\section{Gráfico 6. Gusto por variedad única del español}

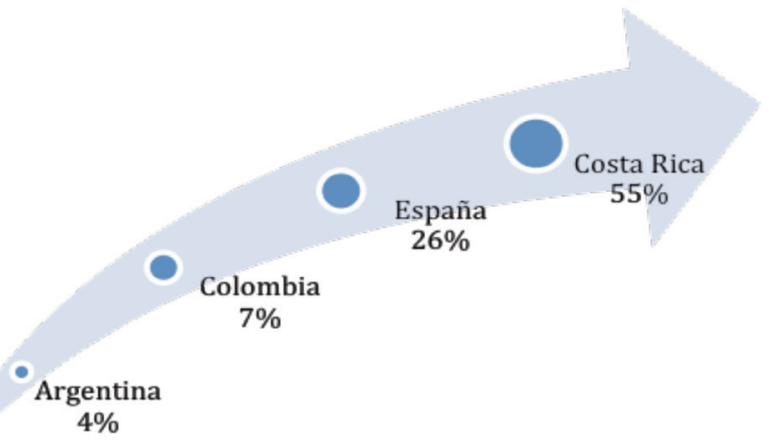

La principal razón por la que se escogió el español de Costa Rica se debe principalmente a que es el español que se habla en el país; mientras que la elección de los otros países se debe principalmente al acento, y en España, "porque se habla el verdadero español". 


\section{Si tuviera que cambiar de acento del español, ¿el de cuál país preferiría?}

De forma similar a la pregunta anterior, el 39\% de los entrevistados indicó que escogería el de Costa Rica, 19\% el de España, 13\% el de Argentina y 11\% el de Colombia. La preferencia por el acento de país no resultó ser estadísticamente significativa por sexo $(p=0,05)$, aunque sí por edad $(\mathrm{p}=0,03)$ explicada porque las personas de 55 años y más indican en mayor proporción que les gusta más la variedad de Costa Rica; también se encontró diferencia estadísticamente significativa por nivel socioeconómico $(\mathrm{p}=0,000)$ ya que las personas de nivel socioeconómico alto tienen preferencia por acento de Costa Rica. Lo mismo sucede con el nivel educativo, en el cual se presentó diferencia estadísticamente significativa $(\mathrm{p}=0,032)$ explicada por la preferencia hacia el acento del país entre los universitarios.

Las características por las que más les gusta el español de España, Colombia y Argentina, es por el acento, porque suena agradable y por la forma de expresarse. No se encontró diferencia estadísticamente significativa según sexo $(\mathrm{p}=0,105)$, nivel socioeconómico $(p=0,089)$ y nivel educativo $(p=0,156)$. Sin embargo, se encontró diferencia estadísticamente significativa por edad $(\mathrm{p}=0,013)$ explicada por la mayor proporción de personas de 35 años y más que indican que les gusta el acento por ser característico del país.

\subsection{El español de otras naciones}

\subsubsection{Percepciones cognitivo-lingüísticas}

Mencione tres países donde hablan el español igual o parecido a como usted lo habla.

Los entrevistados indicaron que los tres países en que se habla parecido a Costa Rica son Colombia (55\%), Panamá (20\%) y El Salvador (19\%).

Gráfico 7

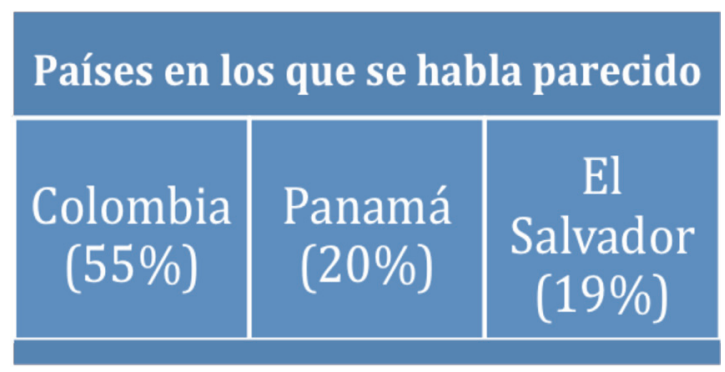

En esta preferencia no se encontró diferencia estadísticamente significativa por sexo ( $p=0,196)$ ni por edad ( $p=0,209)$; se encontró diferencia significativa por nivel socioeconómico. A las personas con bajos ingresos les gusta el español de otros países en menor proporción que a los que se indicaron con más frecuencia. El nivel educativo también resultó ser estadísticamente significativo, ya que se encontró que la proporción de personas que consideran que otros países hablan parecido fue mayor en los que tienen educación primaria o secundaria comparada con los que tienen educación universitaria. Es decir, las personas con educación universitaria tienen mayor capacidad de detectar gente que habla español parecido que las personas que tienen educación primaria o secundaria. 
Mencione tres países donde hablan el español diferente a como usted lo habla.

Para los entrevistados, los tres países en los que se habla diferente son Nicaragua (46\%), Argentina (40\%) y Cuba (25\%).

Gráfico 8. Países en los que se habla diferente

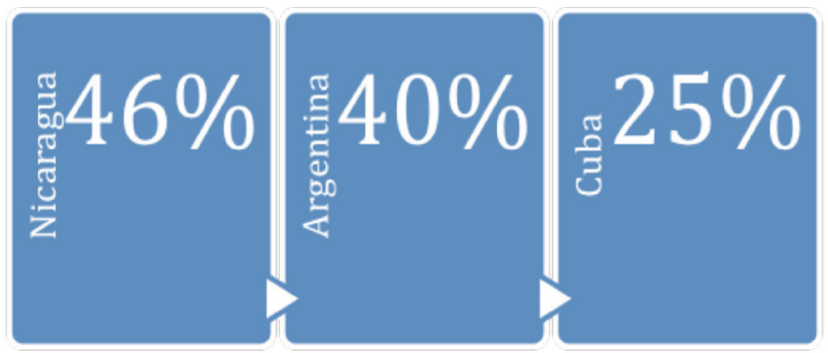

No se encontró diferencia estadísticamente significativa por sexo $(\mathrm{p}=0428=$, edad $(p=0,490)$ o nivel educativo $(p=0,308)$. El nivel socioeconómico sí presenta diferencia estadísticamente significativa $(\mathrm{p}=0,026)$, ya que existe mayor proporción de personas de los niveles socioeconómicos bajo y medio quienes indican que Nicaragua es el país que habla diferente con respecto a las personas de nivel socioeconómico alto.

\subsection{Actitudes afectivas}

\subsubsection{Preferencias generales por variantes dialectales}

Los hablantes muestran preferencia por la manera como se habla en España (53\%), Costa Rica (50\%) y Colombia (47\%). Estos resultados concuerdan en gran medida con los expuestos en las respuestas acerca de en cuáles países se considera que se habla mejor español.

Gráfico 9. Preferencias por variantes dialectales
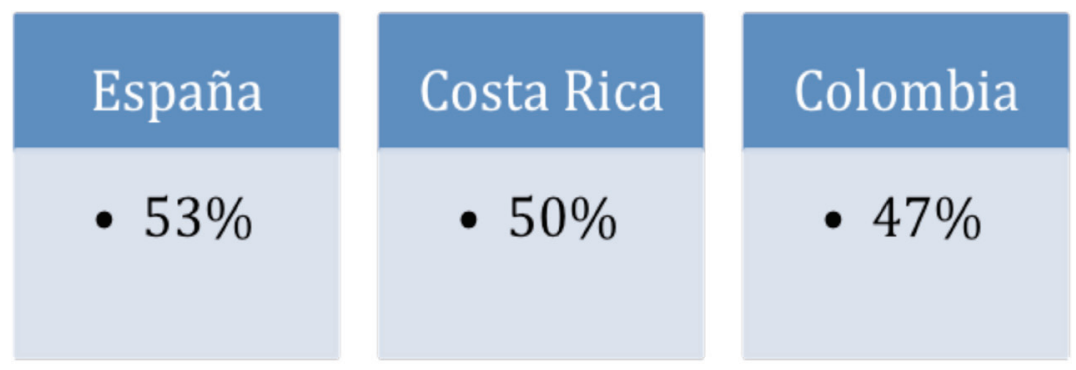

¿Le gustaría que su hijo o hija aprendiera con un/a maestrola o profesor/a originario/a de otro de los países de habla hispana?

Con respecto a la pregunta anterior, el 53\% indicó que no; no se encontró diferencia estadísticamente significativa por edad $(\mathrm{p}=0,882)$, pero sí se encontró diferencia estadísticamente significativa por sexo $(\mathrm{p}=0,03)$, ya que la proporción de hombres que indicó que no, fue de un $60 \%$, mientras que en las mujeres este porcentaje fue de un $45 \%$. Este resultado, al igual que el de la pregunta anterior, ¿Cuán importante considera usted hablar correctamente? establece un paralelo entre sus respuestas en el sentido de la alta importancia que le dan los hombres a la manera de hablar, tanto en la corrección como en la relevancia de no diferenciarse por el 
acento. Cabe recordar que en la pregunta anterior de por qué se considera que en determinados países se habla más correctamente, los hombres le dieron más importancia al "acento". Así, son congruentes entre el hecho de que la importancia en la corrección está en el acento y el no estar de acuerdo en tener profesores o maestros de acento extranjero para sus hijos. De nuevo, aquí se muestra la tendencia a de los hombres hacia percibir la importancia que socialmente se le da a la corrección lingüística y hacia percibir los acentos como más o menos prestigiosos, lo cual se podría traducir en una mayor conciencia sociolingüística.

También se encontró diferencia estadísticamente significativa por nivel socioeconómico $(\mathrm{p}=0,013)$ explicada por la mayor proporción de personas que indican que no les gustaría un maestro de otro país para sus hijos en los niveles socioeconómicos medios y altos (59\%); mientras que en el nivel socioeconómico bajo esta proporción es menor (45\%).

El nivel educativo presentó diferencia estadísticamente significativa; se encontró que las personas con nivel universitario presentan una alta proporción $(\mathrm{p}=0,03)$ de un $62 \%$ que indica que no le gustaría que su hijo recibiera clases con un maestro o profesor extranjero, comparado con el porcentaje observado de $47 \%$ entre las personas con niveles educativos de primaria y secundaria.

En el caso de esta pregunta, el reconocimiento de que hay "acentos" extranjeros y su valoración social corre más a cargo de los sociolectos más altos y más instruidos y de los hombres, quienes en esta investigación se han descubierto como más proclives a la capacidad distintiva de la corrección y de los acentos en relación con una mayor conciencia sociolingüística, lo cual muestra disonancia con el estudio de 1979 de López-Morales para el español de San Juan (Puerto Rico), quien señala que "Las cifras comprueban que las clases medias sobre todo el sexo/género femenino, se muestran muy sensibles a la valoración social de los hechos lingüísticos". Por supuesto, de 1979 a estas fechas han pasado más de treinta años, con lo cual hasta en el mismo Puerto Rico podrían haber cambiado ya los resultados.

\subsubsection{Preferencias dialectales en los medios de difusión}

¿En el español de qué país le gustaría que se dieran las noticias de la radio?

El español preferido para las noticias por radio es el de Costa Rica (67\%), luego España (14\%) y después, Argentina (5\%).

\section{Gráfico 10}

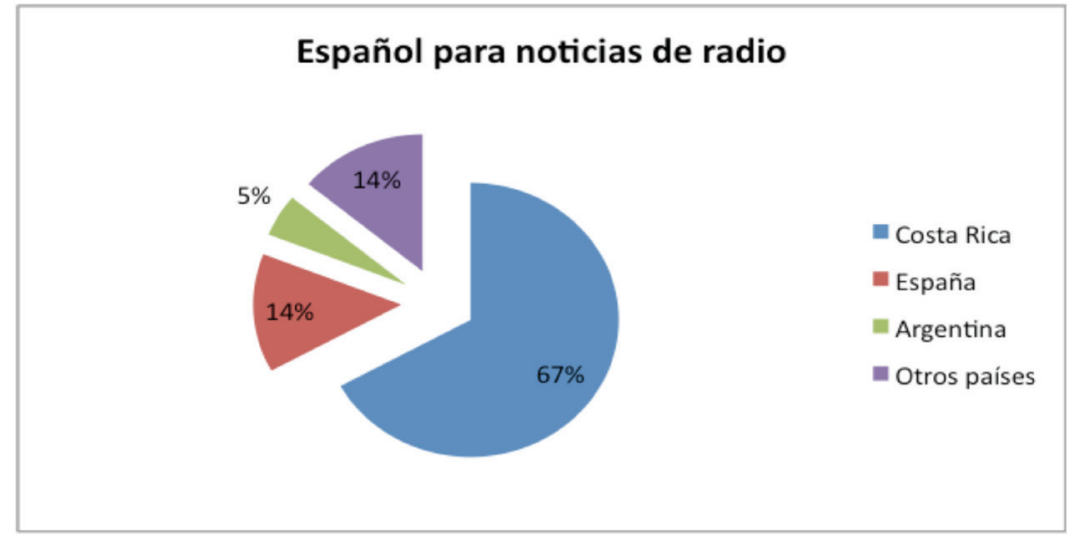


Por grupo de edad se encontró diferencia estadísticamente significativa $(\mathrm{p}=0,036)$, ya que los jóvenes de 20 a 34 años muestran preferencia porque se den las noticias de la radio en español de Costa Rica, mientras que las personas de 55 años y más muestran preferencia hacia el español de España.

La principal razón por la que las personas que indicaron que les gustaría que las noticias de la radio se dieran en español de Costa Rica fue porque el $45 \%$ lo entiende y el 37\% porque es del país, mientras que el español de Argentina fue preferido por el acento (62\%), al igual que el de España en una menor proporción (31\%).

¿En el español de qué país le gustaría que se dieran las noticias de la televisión?

El español preferido en este caso fue el de Costa Rica (64\%), el de España (15\%) y el de México (5\%). La preferencia por el español de Costa Rica se da "porque es del país" (40\%) y "se entiende" (35\%); el de España es preferido por el acento y la pronunciación (35\%) mientras que el México, además del acento y pronunciación (27\%), un 18\% también indica que es "por la forma de expresarse".

¿En el español de qué país le gustaría que le dieran información por teléfono?

En cuanto a la información por teléfono, el $79 \%$ indica que le gusta el español de Costa Rica, el 7\% el español de España.

\section{Gráfico 11}

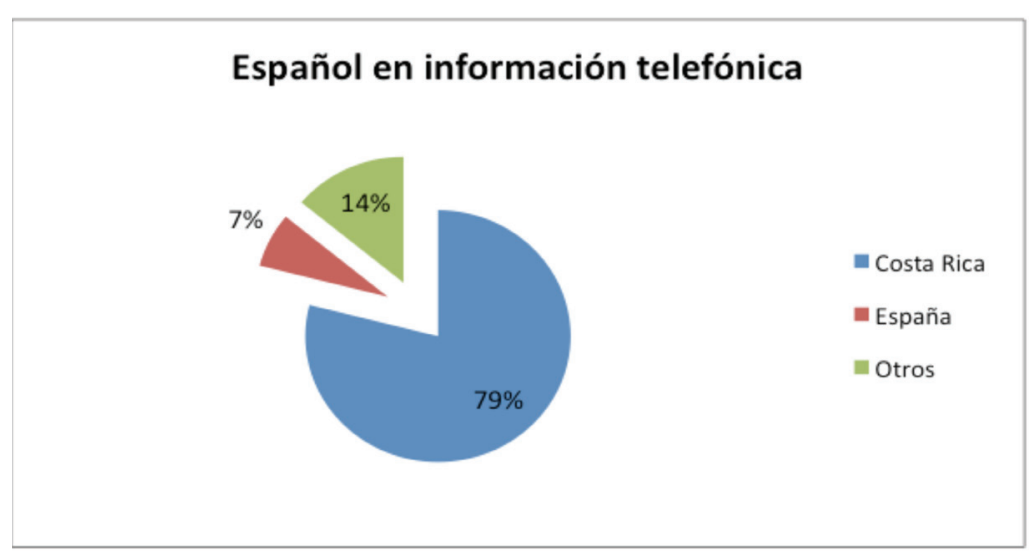

No se encontró diferencia estadísticamente significativa por sexo $(\mathrm{p}=0,782)$, por edad $(\mathrm{p}=0,273)$ ni por nivel educativo $(\mathrm{p}=0,05)$. Se encontró diferencia estadísticamente significativa por nivel socioeconómico $(\mathrm{p}=0,017)$, ya que el estrato medio mostró preferencia hacia el español de Costa Rica, con respecto a la presentada por los estratos bajo y alto.

Las razones por las que a las personas les gusta que la información por teléfono sea con el español de Costa Rica es "porque se entiende" (42\%) y "es del país" (34\%), mientras que a los que les gusta el español de España es simplemente porque les gusta (48\%).

\section{¿En el español de qué país le gustaría que se doblaran las películas?}

El $55 \%$ indica que en el de Costa Rica, un $18 \%$ en el de México y un $7 \%$ en el de España. Se encontró diferencia estadísticamente significativa por nivel socioeconómico, ya que el nivel más alto indica un mayor porcentaje de preferencia por el español de otros países, 
mientras que el estrato medio indica preferencia por el español de Costa Rica; con respecto al nivel educativo $(\mathrm{p}=0,000)$ los universitarios muestran preferencia por el doblaje del español de otros países, y menos el de Costa Rica.

En cuanto a la opinión con respecto a la publicidad de televisión grabada por personas que hablan español de un país diferente al propio, se encontró que al $41 \%$ le es indiferente o la encuentra bien; al $28 \%$ no le gusta y a un $16 \%$ le gusta. Se encontró diferencia estadísticamente significativa por nivel socioeconómico $(\mathrm{p}=0,01)$ y por nivel educativo $(\mathrm{p}=0,01)$, ya que se muestra en los niveles altos y de mayor educación, una preferencia por las categorías "me es indiferente", y menor por las de "no lo entiendo".

\section{Conclusiones} resultados:

Con base en todas las opiniones emitidas, es importante sintetizar los siguientes

a. Para el español general, y sobre "hablar correctamente", los hombres, en su totalidad, consideran muy importante e importante hablar correctamente. En las mujeres hay un pequeño porcentaje que manifiesta que es "poco importante" el cual, sin embargo, es el que explica la diferencia estadísticamente significativa.

b. Para los josefinos, los países en los que se habla más correctamente son España (por mucho, sin que sea el criterio generalizado de los niveles socieconómicos más altos y más educados), Costa Rica, Colombia y Chile.

c. Nicaragua es el país en el cual consideran que se habla más incorrectamente, seguido de México y Puerto Rico. Hay mayor consciencia de que esto no es así en los entre las personas de educación universitaria y las que tienen nivel socioeconómico más alto, por lo que se puede prever una mayor conciencia lingüística en esta población.

d. En cuanto a unidad lingüística, el $70 \%$ preferiría que todos hablaran la misma variedad, principalmente las personas mayores de 55 años y también las personas sin educación universitaria. La razón por la preferencia de la unidad lingüística consiste en el ideal de entenderse mejor. Las personas que no están a favor de la unidad lingüística, indican que se perderían la diferenciación cultural y la idiosincrasia de cada país. En este punto, se consideran de nuevo muy previsivas las palabras de Coseriu (1990) en el sentido de que ni los enfoques muy conservadores ni los muy liberales son adecuados a la hora de percibir la unidad lingüística, y que no es razonable aspirar a una homogeneidad y fijeza total en el plano de las normas; antes bien, la unidad del idioma debe ser panhispánica y dinámica, flexible, política y culturalmente "oportuna".

e. Si se tuviera que hablar el mismo español, el primero sería por mucho el de Costa Rica, por ser el "nuestro"; luego el de España, por ser el "verdadero español”; el de Colombia y el de Argentina. Se perciben actitudes positivas y de identidad relacionadas con la variedad propia.

f. En cuanto a las percepciones cognitivo-lingüísticas, los tres países en los que se habla parecido a Costa Rica son Colombia, Panamá y el Salvador. Los tres países en los que se habla diferente son Nicaragua, Argentina y Cuba. La mayoría de las personas de los niveles socioeconómicos bajo y medio son quienes más indican que Nicaragua es el país en que se habla diferente. 
g. En cuanto a las actitudes afectivas, las preferencias por variantes dialectales son hacia España, Costa Rica y Colombia.

h. Con respecto a si le gustaría que el hijo o hija aprendiera con un/a maestro/a o profesor/a de otro país de habla hispana la mayoría indicó que no, principalmente los hombres, las personas de niveles socioeconómicos medios a altos y las personas universitarias.

i. Sobre las preferencias dialectales en los medios de difusión, el español preferido para las noticias por radio son Costa Rica (67\%), España (14\%) y Argentina (5\%). El español preferido para las noticias de la televisión fue el de Costa Rica (64\%), el de España (15\%) y el de México (5\%). En cuanto a dar información por teléfono, el 79\% indica que le gusta el español de Costa Rica y el 7\% el español de España. Para el doblaje de películas, la preferencia es hacia el español de Costa Rica, 55\%, el de México, un 18\%, y el de España, $7 \%$.

Los datos anteriores revelan que los estratos más altos de la sociedad costarricense, por educación y por nivel socioeconómico, demuestran mayor conciencia sociolingüística; que los hombres, en algunos rubros más que las mujeres, presentan mayor conciencia acerca de la corrección y el prestigio linguiísticos; que ellas son más proclives a la innovación; que los estereotipos sobre los países se reflejan también en los estereotipos lingüísticos: desde el gusto y el disgusto por su forma de hablar, el español más correcto, el "verdadero" y el preferido, se sigue asociando a España, y el más incorrecto a Nicaragua. Costa Rica presenta niveles bastante altos de aceptación y de actitudes afectivas positivas, en contraste con otros estudios de actitudes desarrollados en el país.

Ante la pregunta final de López-Morales (2005) ¿Qué español hablaremos? Para el español de Costa Rica se podría seguir diciendo que el español de Costa Rica, quizás con menos colorido local, sin tantos giros o expresiones regionales como antaño, y siempre con la añoranza del "correcto español" que sigue siendo idealmente el de España. Los medios como internet, la televisión por cable o por satélite y la facilidad que existe en la actualidad para viajar a otros países, nos expone con mayor facilidad a las diversas variedades, lo cual resulta positivo pues, como señala López-Morales (2005), esto permite ampliar la nómina pasiva del léxico de un hablante de español. Por otro lado, la unidad y la homogeneidad del español, parecieran estar aseguradas:

\footnotetext{
Estudios lexicoestadísticos muy solventes nos demuestran una y otra vez que existe una unidad sobresaliente entre las diversas variedades de nuestra lengua. Juan Miguel Lope Blanch (2000), en un recuento de 133.000 vocablos seleccionados del habla de Madrid, descubrió que el 99,9\% era vocabulario común a México; las que disonarían en ese país hispanoamericano alcanzaban apenas un 0,1\% del total. En resumen, que Madrid y México coinciden casi en un $100 \%$ con el vocabulario de la norma culta del español general. (López-Morales, 2005, p. 486)
}

\section{Notas}

1. El instrumento utilizado en la investigación es una elaboración colectiva de los participantes en el proyecto LIAS de la Universidad de Bergen (2009-2012). Los resultados de este estudio se pueden encontrar en Chiquito y Quesada-Pacheco (2014), https://bells.uib.no/.

2. "Cuando Bello se decidió a escribir su Gramática le guiaron dos móviles esenciales: el primero se dirigía a preservar y mantener en su posible unidad el idioma de nuestros mayores y antepasados; el segundo, 
se encaminaba a evitar que tuviera un proceso de desintegración parecido al latín al desmembrarse el Imperio Romano, surgiendo en su antiguo habitat las lenguas conocidas como neolatinas." (UrrutiaCárdenas, 1992, p. 254).

3. Bello, A. (1847). Gramática de la lengua castellana destinada al uso de los americanos. Santiago de Chile: Imprenta del Progreso.

4. Sin página, documento electrónico.

\section{Bibliografía}

Bello, A. (1847).Gramática de la lengua castellana destinada al uso de los americanos. Santiago de Chile: Imprenta del Progreso.

Calvo Shadid, A. y Castillo-Rivas, J. (2014). Las actitudes lingüísticas en el español de San José, Costa Rica. Por A.B. Chiquito y M.Á. Quesada-Pacheco (Eds.). Actitudes lingüísticas de los hispanohablantes hacia el idioma español y sus variedades. (Vol. 5). (249-289). Universidad de Bergen: BeLLS.

Chiquito, A.B. y Quesada-Pacheco, M.Á. (Eds.). Actitudes lingüísticas de los hispanohablantes hacia el idioma español y sus variedades. [versión digital]. Universidad de Bergen: BeLLS. https://bells.uib.no/

Coseriu, E. (1990). El español de América y la unidad del idioma. I Simposio de filología iberoamericana. Universidad de Sevilla. (43-75).

Instituto Cervantes. (2009). Guía práctica del español correcto. Madrid: Espasa Libros.

Instituto Cervantes. (2012). El libro del español correcto. Madrid: Espasa Libros.

Instituto Cervantes. (2013). Las 500 dudas más frecuentes del español. Madrid: Espasa Libros.

López-Morales, H. (1979). Dialectología y sociolingüística: Temas puertorriqueños. Madrid: Hispanova de Ediciones.

López-Morales, H. (2004). Sociolingüística. Madrid: Gredos.

López-Morales, H. (2005). El futuro del español. [pdf.]. http://cvc.cervantes.es/ [Consulta: 14 de febrero de 2014].

Martínez de Sousa, J. (2005). El Diccionario panhispánico de dudas, ¿cumple con su deber? [pdf.]. http://www.martinezdesousa.net/ [Consulta: 08 de febrero de 2014].

Real Academia Española de la Lengua y Asociación de Academias de la Lengua Española. (2005). Diccionario Panhispánico de Dudas. Bogotá: Santillana.

Real Academia Española de la Lengua y Asociación de Academias de la Lengua Española. (2012). El buen uso del español. Madrid: Espasa.

Urrutia-Cárdenas, H. (1992). Andrés Bello: la unidad y variedad del español. CAUCE. (14, 15), 253-262.

Wagner, C. (2006). Andrés Bello y la Gramática Castellana latinoamericana. Documentos Lingüísticos y Literarios. 29. http://www.humanidades.uach.cl/ [Consulta: 17 de febrero de 2014]. 